\title{
Comparison of Treadmill and Kayak Ergometer Protocols for Evaluating Peak Oxygen Consumption
}

\author{
José Augusto Rodrigues dos Santos ${ }^{1,2, *}$, Rui Filipe Santos Sousa ${ }^{1}$ and Tânia Patrícia Amorim ${ }^{1,2}$ \\ ${ }^{1}$ University of Porto, Faculty of Sport, Portugal \\ ${ }^{2}$ Centre of Research, Education, Innovation and Intervention in Sport (CIFI2D- FADEUP), Portugal
}

\begin{abstract}
The main goal for training in sport is to elicit the adequate physical loads to induce the desired adaptations. To achieve this goal it is necessary to continuously assess the alterations induced by training, which can be done by field or laboratory tests. The aim of the present study is to compare peak oxygen consumption of elite kayakers on treadmill and kayak ergometer protocols in two moments of a training season. Five elite sprint kayakers performed two maximal tests (treadmill and kayak ergometer) to assess peak oxygen consumption, maximum heart rate, and performance indicators. Tests were conducted in two different moments of the season: in the preparatory period in October (M1), and in the competitive period in April (M2). Peak oxygen consumption on treadmill were 4.66 \pm 0.44 L.min-1 (58.4 \pm 3.3 ml.kg-1.min $\left.{ }^{-1}\right)$ and 4.55 \pm 0.31 L.min-1 $\left(58.0 \pm 3.2 \mathrm{ml} . \mathrm{kg}-1 . \mathrm{min}^{-1}\right)$ in M1 and M2, respectively; on kayak ergometer the values for this pa-

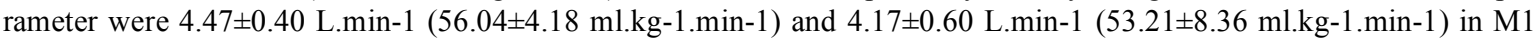
and M2, respectively. The statistical analyses (Friedman Test and Test $\mathrm{T}$ Wilcoxon) showed no significant differences $(p>0.05)$ between ergometers in the two moments of the study. The data obtained, both in treadmill and kayak ergometer, also showed no significant differences $(p>0.05)$ between moments of a training season. It was concluded that in well trained kayakers the achievement of peak oxygen consumption is similar using treadmill or kayak ergometer exercise, and that this parameter is not suitable to detect performance alterations during the season.
\end{abstract}

Keywords: Kayaking, kayak ergometer, treadmill, VO2peak.

\section{INTRODUCTION}

Systematic evaluation is crucial for training control, and testing peak oxygen consumption (VO2peak) can be relevant for training control in sports that depend on high aerobic capability [1]. Running and cycling ergometers are normally used to assess oxygen consumption, even in athletes enlisted in sport modalities eliciting different biomechanical patterns [2].

Sprinter kayakers should develop high aerobic capacity to respond to the high metabolic demands promoted by flatwater racing distances. VO2peak assessment in kayakers was the goal for several studies [e.g. 3-5] highlighting the stress imposed in aerobic metabolism during competition distances, mainly 500-m and 1000-m [6]. VO2peak in kayakers is usually assessed with arm crank or treadmill ergometers [5], which impair the specificity of muscle recruitment [7]. Although the development of specific ergometers for kayakers began earlier in the 1970s $[8,9]$ their worldwide utilization started some years later. In experienced kayakers, it was determined that arm crank ergometer elicited a significant lower relative and absolute VO2peak, when compared with kayak ergometer $\left(44.2 \pm 6.2 \mathrm{ml} \cdot \mathrm{kg}^{-1} \cdot \mathrm{min}^{-1}\right.$ and $3.14 \pm 0.64$ L. $\mathrm{min}^{-1}$ versus $47.5 \pm 3.9 \mathrm{ml} \cdot \mathrm{kg}^{-1} \cdot \mathrm{min}^{-1}$ and $3.38 \pm 0.53 \mathrm{~L} \cdot \mathrm{min}^{-1}$ ) [7].

*Address correspondence to this author at the Rua Dr. Plácido Costa, 91 4200.450 Porto. Portugal; Tel: 00351220425200; Fax: 00351225500689;

E-mail: jaugusto@fade.up.pt
These differences can be related to greater muscle mass involved on the kayak ergometer or to the inadequacy of the crank movement to mobilize efficiently all the muscles of trunk and arms. In fact, crank ergometer testing neglects the important contribution of the legs for kayaking technique, as kayaking involves important legs' actions what may justify rationality for running training imposed by some coaches, at least during the winter phase of the season. Moreover, it was found that maximal oxygen uptake (VO2max) with the arms corresponds to $88.6 \%$ of the value attained with legs [10], which also justify running training as a mean to develop a cardiovascular reserve, eventually utilized in kayaking.

Therefore, the aim of the present study was to test, in elite kayakers, if there are differences between VO2peak and heart rate peak (HRpeak) values obtained with different laboratory devices (treadmill and kayak ergometer), and if the eventual differences are maintained throughout the season.

\section{METHODS}

\section{Subjects}

Five elite sprint kayakers (age 19.6 \pm 2.7 years; height $179.9 \pm 3.7 \mathrm{~cm}$ ) with $6.2 \pm 2.4$ years of training and kayaking competition participated in this study. All the paddlers were used to running on a treadmill and to paddle in the kayak ergometer. The experimental procedures and the possible risks involved in the study were explained to the paddlers who provided prior written. The study protocol was ap- 
proved in advance by the Ethics Committee of the Scientific Board of Faculty of Sport, University of Porto, and was designed in accordance to the recommendations of the Declaration of Helsinki.

\section{Testing Procedures}

In two moments of the season, the first in the preparatory period in October (M1) and the second within the competitive period in April (M2), the paddlers performed two maximal tests, one in a treadmill and the other in a kayak ergometer, to assess VO2peak, HRpeak, and performance indicators. Anthropometric measurements included: body mass and skinfold thickness (triceps brachii, biceps brachii, subscapular, and suprailiac). All the measurements were made by the same experienced technician in accordance with the procedures proposed previously [11]. Height was assessed to the nearest $0.1 \mathrm{~cm}$ (CHARDER HM 200 P Portstad Portable Stadiometer) and body mass to the nearest $0.1 \mathrm{~kg}$ (SECA Robusta 813 High Capacity Digital Floor Scale). Skinfold thickness was assessed using a skinfold caliper, accurate to $0.2 \mathrm{~mm}$ (LANGE - Harpenden; Holtain Ltd., UK). Body density and percentage of body fat were estimated by equations presented in the literature [13, 14]. Subjects did not alter significantly their body mass (M1: $80.3 \pm 7.2 \mathrm{~kg}$; M2: $78.7 \pm 7.2 \mathrm{~kg}$, and body fat (M1: $10.7 \pm 4.8 \%$; M2: $9.7 \pm 3.6$ $\%)$. After one day of complete rest, the incremental running test was performed on a treadmill (Quassar-Med, Nussdorf, Germany), with $8 \mathrm{~km} \cdot \mathrm{h}^{-1}$ of starting velocity, a speed increase of $2 \mathrm{~km} \cdot \mathrm{h}^{-1}$ every two min until exhaustion, and a $0 \%$ slope. $24 \mathrm{~h}$ latter, the incremental paddling test was performed on an air-braked kayak ergometer (K1 ERGO, Garran, Australia), which was interfaced with a computer for measuring performance data, and has previously been shown to accurately simulate the physiological demands of open water kayaking [14]. The subjects started paddling with an initial load of $120 \mathrm{~W}$ with increments of $30 \mathrm{~W}$ every two min until exhaustion. Subjects were asked to perform their maximum and were verbally encouraged by the lab technicians and the team coach. All the participants reached at least two of the following criteria at the end of the test: VO2 plateau, respiratory quotient above 1.1 or subjective feeling of maximum effort with incapability to continue the task [15].

Expired respiratory gas fractions were measured using an open circuit breath-by-breath automated gas analysis system (Cortex, Metalyzer, 3B, Leipzig, Germany). Device calibration was performed using gas from a bottle with the reference of $15 \% \mathrm{O} 2$ and $5 \% \mathrm{CO} 2$. Before each test, volume (turbine) was calibrated using a $3 \mathrm{~L}$ syringe. HR was measured and recorded every $5 \mathrm{~s}$ using a HR monitor (Vantage NV, Polar Electro, Kempele, Finland) that was connected with the gas analyzer system.

\section{Statistics Procedures}

Descriptive statistics (mean \pm standard deviation) were determined for all variables. The physiological and performance variables assessed were compared between moments by Friedman test. The differences between ergometers in the two moments were compared by the Test $\mathrm{T}$ Wilcoxon. An alpha level of $p<0.05$ was chosen as the criterion for statistical significance for all comparisons.

\section{RESULTS}

Physiological and performance data obtained in treadmill showed no significant differences between moments (Table 1), but there was a trend to lower VO2peak in M2. The data obtained in the kayak ergometer also did not showed any significant differences between moments (Table 1).

In adittion, the absence of significant changes in VO2peak between ergometers in the two moments of the study is expressed in Table $\mathbf{2}$.

\section{DISCUSSION}

The aim of this study was to test the differences in VO2peak and HRpeak obtained by two different protocols treadmill and kayak ergometer. Running is a fundamental issue for paddler training at least in winter season when cross-training is widely used. As the specific VO2max of exercising using only the arms is usually lower than that attained with legs $[9,10]$, endurance legs' exercises, putting an additional overload on the cardiovascular system, can contribute to improve cardiac output during kayaking. However, conflicting with our proposal, transfer effects from legs to arms seem to be ineffective because cardiovascular adaptations are specific to the muscle groups that are trained [16]. Endurance training enhancement can be both specific (peripheral adaptations) and non-specific, probably due to central adaptations [17]. As legs' work is a fundamental part of the biomechanical stress imposed by paddling, it is supposed that some benefits for paddling can result from running training. Supported in these assumptions, it was expected that treadmill protocol could elicit higher VO2peak and HRpeak that kayak ergometer protocol, but the current results did not corroborate this assumption. Actually, this study showed that in M1 (preparatory phase of the season), VO2peak (absolute and relative), and HRpeak values were similar, as no differences between treadmill and kayak ergometer protocols were observed. This similarity can be justified by the percent of muscle mass mobilized with the kayaking ergometer that seems to be enough to promote identical VO2peak in treadmill or due to a superior technical efficiency expressed by the elite kayakers that outstrip the eventual mechanical limitations imposed by the kayak ergometer. Our results showed that elite kayakers did not reached a higher VO2peak with legs' ergometers, probably due to the specificity of kayaking training. Physiological parameters in kayakers are similar to untrained subjects when obtained in non-specific ergometers and similar to other highly trained athletes when achieved by specific ergometers [18]. In M2 (competitive phase of the season), the differences between the two protocols continue to have no statistical significance. However, VO2peak values, slightly lower (as a tendency) for kayak ergometer in both moments, are in accordance with the literature [14], who showed that $\mathrm{VO} 2$ peak in kayaking corresponded to 80 $100 \%$ of VO2peak in running.

For each ergometric device the differences in VO2peak between M1 and M2 were not statistically significant $(p>0.05)$. VO2peak values in this study were similar to the results obtained by other authors ranging from 3.15 to 5.15 L. $\min ^{-1}$ and $54-60 \mathrm{ml} \cdot \mathrm{kg}^{-1} \cdot \mathrm{min}^{-1}[3,5,12,19,20,21]$, but markedly lower than the obtained by elite Spanish flatwater kayakers $\left(61.1 \pm 2.7\right.$ to $\left.68.6 \pm 3.2 \mathrm{ml} . \mathrm{kg}^{-1} \cdot \mathrm{min}^{-1}\right)$ [5]. The best 
Table 1. Physiological and Performance Data in Treadmill and in Kayak Ergometer

\begin{tabular}{|c|c|c|c|}
\hline Variables & Moment 1 & Moment 2 & $\mathbf{p}$ \\
\hline \multicolumn{4}{|c|}{ Treadmill } \\
\hline VO2peak $\left(\right.$ L.min $\left.{ }^{-1}\right)$ & $4.66 \pm 0.44$ & $4.55 \pm 0.31$ & 0.080 \\
\hline VO2peak(ml.kg-1.min-1) & $58.4 \pm 3.3$ & $58.0 \pm 3.2$ & 0.686 \\
\hline HRpeak (b.min-1) & $188.8 \pm 10.0$ & $185.2 \pm 11.3$ & 0.458 \\
\hline RERpeak & $1.08 \pm 0.04$ & $1.12 \pm 0.07$ & 0.686 \\
\hline RSpeak (m.s-1) & $5.58 \pm 0.4$ & $5.58 \pm 0.4$ & 1 \\
\hline Dpeak (m) & $2728 \pm 489$ & $2963 \pm 541$ & 0.138 \\
\hline \multirow[t]{2}{*}{ TPeak (s) } & $787.6 \pm 99.2$ & $826 \pm 118$ & 0.138 \\
\hline & Kayak ergometer & & \\
\hline VO2peak (L.min-1) & $4.47 \pm 0.40$ & $4.17 \pm 0.60$ & 0.345 \\
\hline VO2peak (ml.kg-1.min-1) & $56.04 \pm 4.18$ & $53.21 \pm 8.36$ & 0.500 \\
\hline HRpeak (beats.min-1) & $182.6 \pm 10.6$ & $181.4 \pm 12.5$ & 0.416 \\
\hline RERpeak & $1.03 \pm 0.09$ & $1.09 \pm 0.06$ & 0.500 \\
\hline PSpeak (m.s-1) & $4.2 \pm 0.1$ & $4.2 \pm 0.2$ & 0.577 \\
\hline Dpeak (m) & $2728 \pm 489$ & $2963 \pm 541$ & 0.138 \\
\hline
\end{tabular}

RSpeak - running speed at VO2peak; Dpeak - distance covered at VO2peak; Tpeak - time spent to achieve VO2peak

Table 2. Comparison Between Ergometers in The Two Moments of The Season in Relation to VO2peak

\begin{tabular}{|l|c|c|}
\hline & Test T Wilcoxon & p \\
\hline \hline Treadmill/kayak ergometer $(\mathrm{L} \cdot \mathrm{min}-1$ in M1) & 0.72 & 0.490 \\
\hline Treadmill/kayak ergometer $\left(\mathrm{ml} \cdot \mathrm{kg}^{-1} \cdot \mathrm{min}^{-1}\right.$ in M1) & 0.98 & 0.520 \\
\hline Treadmill/kayak ergometer $\left(\mathrm{L} \cdot \mathrm{min}^{-1}\right.$ in M2) & 1.25 & 0.250 \\
\hline Treadmill/kayak ergometer $\left(\mathrm{ml} \cdot \mathrm{kg}^{-1} \cdot \mathrm{min}^{-1}\right.$ in M2) & 1.12 & 0.320 \\
\hline
\end{tabular}

paddler in our study showed a relative lower VO2peak (4.72 L. $\mathrm{min}^{-1}$ and $55.8 \mathrm{ml} \cdot \mathrm{kg}^{-1} \cdot \mathrm{min}^{-1}$ ), but got a 6th place in the final of the 1000-m race in the Olympic Games, 3 months after M2. Although flatwater kayaking performance (mainly $500-\mathrm{m}$ and $1000-\mathrm{m}$ races) is highly supported by aerobic metabolism, it does require a large anaerobic contribution [19] what reduces the absolute importance of the aerobic energetic pathway. Moreover, enhancement of performance in laboratory or field tests verified in most studies may not apply to elite athletes in competitive events [22].

Body mass changes, even without statistical significance $(p=0.273)$ decreased slightly concurrently with the slight decrease of fat mass. Elite athletes tend to remain active during the transition period between seasons avoiding dramatic increases in fat mass. In elite athletes body mass variance over the season is commonly low [4], unless dramatic increasing of training volume is introduced in the training programs [23]. Therefore, in this study variations in VO2peak may be partially explained by body mass alterations.

Treadmill and kayak ergometer performances were not statistically different between moments $(p=0.138)$, albeit showing a trend for enhancement. The stability of the laboratory results throughout the season strengths the statement of Hopkins et al. [23], which put in question the validity of the laboratory and field tests to assess performance in competi- tive events. The subjects of the present study achieved average paddling powers at VO2peak higher than those obtained by García-Pallarés et al. [5] with elite Spanish kayakers in different moments of the season. These marked differences can be attributed to methodological procedures (e.g. different protocols or different kayak ergometers) or exercise strategies. Strengthen this assumption, after a 2-min kayak ergometer test with different starting strategies Bishop et al. [19] verified, with the same athletes, that an all-out start achieved a significant higher $(\mathrm{p}<0.05)$ peak power $(747 \pm 151$ $\mathrm{W})$ in relation to an even start $(558 \pm 110 \mathrm{~W})$.

In the present study, HRpeak was lower in kayak ergometer in relation to the treadmill, but was only significant $(\mathrm{p}=0.04)$ in $\mathrm{M} 1$. Arms ergometers testing seem to elicit lower HRmax than legs ergometers [17] what can be related to muscular constraints. Our data permit to verify similar VO2peak achieved with different heart rate peaks what highlights the specificity of cardiac response to each ergometer.

\section{CONCLUSION}

The reduced number of subjects and their performance homogeneity do not allow us to build strong and unquestionable conclusions. However, elite kayakers achieved similar VO2peak with kayak ergometer and treadmill, which seems to be related to the importance of running in the training pro- 
grams for kayakers. Moreover, muscle legs are importantly activated during paddling in highly skilled paddlers what can contribute also for the similitude of results. As kayak ergometer and treadmill performances are stable between moments, they did not allow assessing the outcome of the training program throughout the season. It was concluded that the selected protocols are not suitable for evaluating physiological alterations throughout the season in elite kayakers.

\section{CONFLICT OF INTEREST}

The authors confirm that this article content has no conflicts of interest.

\section{ACKNOWLEDGEMENT}

Declared none.

\section{REFERENCES}

[1] Thoden J. Physiological testing of the high-performance athlete. In: MacDougall JD, Wenger HA, Green HJ, Eds. Champaign IL: Human Kinetics 1991; pp. 107-73.

[2] Fukuda D, Smith A, Kendall K, Cramer J, Stout J. The determination of critical rest interval from the intermittent critical velocity test in club-level collegiate hockey and rugby players. J Strength Cond Res 2011; 25(4): 889-95.

[3] Fry R, Morton A. Physiological and kinanthropometric attributes of elite flatwater kayakists. Med Sci Sports Exerc 1991; 23: 1297-301.

[4] García-Pallarés J, García-Fernández M, Sánchez-Medina L, Izquierdo M. Performance changes in world-class kayakers following two different training periodization models. Eur J Appl Physiol 2010; 110: 99-107.

[5] Tesch P, Piehl K, Wilson G, Karlsson J. Physiological characteristics of elite kayak paddlers. Can J Appl Spot Sci 1083; 8(2): 87-91.

[6] Shephard R. Science and medicine of canoeing and kayaking. Sports Med 1987; 4: 19-33.

[7] Forbes S, Chilibeck P. Comparison of a kayaking ergometer protocol with an arm crank protocol for evaluating peak oxygen consumption. J Strength Cond Res 2007; 21(4): 1282-5.

[8] Pyke F, Baker J, Hoyle R, Scrutton E. Metabolic and circulatory responses to work on a canoeing and bicycle ergometer. Am J Sports Med 1973; 5: 22-31.
[9] Zavgorodniy U. Device for training kayak rowers during the winter. Yessis Rev Soviet Phys Educ Sports 1978; 13: 2.

[10] Vrijens J, Hoekstra P, Bouckaert J, Van Uytvanck P. Effects of training on maximal working capacity and haemodynamic response during arm and leg-exercise in a group of paddlers. Eur J Appl Physiol Occup Physiol 1975; 34: 113-9.

[11] Ross W, Marfell-Jones M. Physiological testing of the elite athlete. In: MacDougall JD, Wenger HA, Green HJ, Eds. New York: Movement Publication 198; pp. 75-115.

[12] Durnin J, Womersley J. Body fat assessed from total body density and its estimation from skinfold thickness: measurements on 481 men and women aged from 16 to 72 years. Br J Nutr 1974; 32(1): 77-97.

[13] Siri W. Techniques for measuring body composition. In: Brozek J, Hensche A, Eds. Washington DC: National Academy of Sciences, National Research Council 1961; pp. 223-44.

[14] Van Someren K, Phillips G, Palmer G. Comparison of physiological responses to openwater kayaking and kayak ergometry. Int $\mathbf{J}$ Sports Med 2000; 21: 200-4.

[15] Rowland TW. Exercise testing. In: Rowland TW, Ed. Development Exercise Physiology. Champaign IL: Human Kinetics 1996; pp. 2747.

[16] Bhambhani Y, Eriksson P, Gomes P. Transfer effects of endurance training with the arms and legs. Med Sci Sports Exerc 1991; 23(9): 1035-41.

[17] Pogliaghi S, Terziotti P, Cevese A, Balestreri F, Schena F. Adaptations to endurance training in the healthy elderly: arm cranking versus leg cycling. Eur J Appl Physiol 2006; 97: 723-31.

[18] Bunc V, Heller J. Ventilatory threshold and work efficiency on a bicycle and paddling ergometer in top canoeists. J Sports Med Phys Fitness 1991; 31(3): 376-9.

[19] Bishop D, Bonetti D, Dawson B. The influence of pacing strategy on VO2 and supramaximal kayak performance. Med Sci Sports Exerc 2002; 34(6): 1041-7.

[20] Van Someren K, Oliver J. The efficacy of ergometry determined heart rates for flatwater kayak training. Int J Sports Med 2002; 23: 28-32.

[21] Billat V, Faina M, Sardella F, et al. A comparison of time to exhaustion at $\mathrm{VO} 2 \mathrm{max}$ in elite cyclists, kayak paddlers, swimmers and runners. Ergono 1996; 39(2): 267-77.

[22] Hopkins W, Hawley J, Burke L. Design and analysis of research on sport performance enhancement. Med Sci Sports Exerc 1999; 31(3): 472-85.

[23] Dos Santos R J. Physiological and jumping performance alterations induced by a dramatic increase of running volume. A case study. Cultura Ciencia Deporte 2011; 18(6): 193-8.

(C) Santos et al.; Licensee Bentham Open.

This is an open access article licensed under the terms of the Creative Commons Attribution Non-Commercial License (http://creativecommons.org/licenses/ by-nc/3.0/) which permits unrestricted, non-commercial use, distribution and reproduction in any medium, provided the work is properly cited. 